\section{Ashkenazi carrier screening for reproductive planning: is this what we planned for?}

To the Editor: In their article entitled "Expanded Genetic Screening Panel for the Ashkenazi Jewish Population," Baskovich et al. ${ }^{1}$ discuss the need "to reframe our goals of population screening and genetic counseling." They propose to use an expanded panel in which the screening is based on clinical utility and frequency of the mutations, not on their disease implications. While there is a need to revise the screening offered to Ashkenazi Jews, in our opinion it is problematic to include in a single panel various genes that predispose to disease and cancer together with mutations leading to diseases relevant to reproductive risk. The Tay-Sachs carrier-screening program aimed to prevent a lethal disease that was frequent in the Ashkenazi Jewish community. Later, with the gradual ability to detect carriers of additional frequent monogenic disorders among Ashkenazi Jews, further tests were added to the carrier-screening programs. Although some of the added disorders did not always adhere to the same principles that were at the basis of the screening program (particularly with respect to criteria such as severity and frequency), the aim remained to enable couples to prevent the birth of affected children. Following the characterization of founder mutations that increase the risk for breast and ovarian cancers, the importance of presymptomatic testing became evident. The possibility of population screening for these mutations was raised, and several studies have been performed in the past decade. ${ }^{2}$ Up to now, however, the vast majority of Ashkenazi Jews performing screening genetic tests are doing so to determine their reproductive risk; relatively few are using them for screening before symptoms occur.

It is our opinion that the default panel offered to Ashkenazi Jews should include mutations known to cause severe diseases. Information on the possibility to test for mutations causing mild phenotypes and/or presymptomatic detection should be offered to screened individuals as an additional option following genetic counseling.

Moreover, Baskovich et al. ${ }^{1}$ designed a screening panel that includes mutations reported in healthy individuals. Among the 128 healthy Ashkenazi Jews in the study, variants were characterized in 40 genes that have not been reported among Ashkenazi Jewish patients. As an example, Baskovich et al. included in their expanded panel "bronchiectasis with or without elevated sweat chloride 2" due to a recessive mutation p.W516R (rs5742912) in SCNN1A. Two submissions for rs5742912 have been reported in ClinVar; the variant was considered pathogenic in one case and benign in the other, and it is classified as having "conflicting interpretations of pathogenicity." ${ }^{3}$ The frequency of this variant among the 128 healthy Ashkenazi Jews who were fully sequenced (0.0195), as reported by Baskovich et al., ${ }^{1}$ also raises the possibility that it is benign given that no homozygous Ashkenazi Jewish patients have been reported. Such variants should not be included in a screening panel until their pathogenicity is proven.

\section{DISCLOSURE}

The authors declare no conflict of interest.

\section{Joël Zlotogora, $\mathrm{MD}, \mathrm{PhD}^{1}$ and Vardiella Meiner, $M D^{1,2}$}

${ }^{1}$ Hadassah Medical School, Hebrew University, Jerusalem, Israel; ${ }^{2}$ Department of Genetics and Metabolic Diseases, Hadassah-Hebrew University Medical Center, Jerusalem, Israel. Correspondence: Joël Zlotogora (zlotogora@gmail.com)

\section{REFERENCES}

1. Baskovich B, Hiraki S, Upadhyay K, et al. Expanded genetic screening panel for the Ashkenazi Jewish population. Genet Med; e-pub ahead of print 3 September 2015.

2. Gabai-Kapara E, Lahad A, Kaufman B, et al. Population-based screening for breast and ovarian cancer risk due to BRCA1 and BRCA2. Proc Natl Acad Sci USA 2014;111:14205-14210.

3. National Center for Biotechnology Information. ClinVar information for NM_001038.5(SCNN1A):c.1477T>C (p.Trp493Arg). http://www.ncbi.nlm.nih. gov/clinvar/variation/9269. Accessed 8 November 2015.

Advance online publication 10 December 2015. doi:10.1038/gim.2015.169 